\title{
Mathematical Programming and Electricity Markets
}

\author{
Antonio J. Conejo \\ Departamento de Ingeniería Eléctrica, ETSI Industriales \\ Universidad de Castilla - La Mancha \\ Campus Universitario s/n, $130 \% 1$ Ciudad Real, Spain \\ e-mail: aconejo@ind-cr.uclm.es \\ Francisco J. Prieto \\ Departamento de Estadística y Econometría \\ Universidad Carlos III de Madrid \\ C/ Madrid, 126, 28903 Getafe (Madrid), Spain \\ e-mail: fjp@est-econ.uc3m.es
}

\begin{abstract}
The electric power industry in Europe and all over the world is undertaking major regulatory and operational changes. The underlying rationale behind all these changes is to move from a centralized operation approach to a competitive one. That is, the understanding of power supply as a public service is being replaced by the notion that a competitive market is a more appropriate framework to supply reliable and cheap electric energy to consumers. In some cases, the aforementioned transition process has included the privatization of power utilities. This new framework requires new tools and procedures, and some of these procedures drastically differ from traditional ones. Therefore, new challenging mathematical programming and operations research problems naturally arise in this context. This paper provides a review of some of these problems, particularly operational problems spanning a time horizon from one day to one year. The approach adopted emphasizes mathematical programming issues, describing the structure and characteristics of these problems and suggesting appropriate solution techniques.
\end{abstract}

Key Words: Electric power, competitive markets, large-scale optimization, mixed-integer optimization.

AMS subject classification: 90B30, 90C06, 90C11, $91 \mathrm{~B} 26$.

\section{Introduction}

The electric power industry in Europe and all over the world is undertaking major regulatory and operational changes. The underlying rationale behind all these changes is to move from a centralized operation approach

Relevant comments and suggestions were provided by Alexis Motto from McGill University, Montréal, Québec, Canada. 
to a competitive one. That is, the understanding of power supply as a public service is being replaced by the notion that a competitive market is a more appropriate framework to supply reliable and cheap electric energy to consumers.

This new competitive paradigm is implemented via two market structures: (i) a power pool and (ii) a floor to facilitate bilateral contracts among producers and consumers.

A pool is an e-commerce marketplace where producers and consumers submit production and consumption bids, respectively. The market operator clears the market using market rules and produces a market-clearing price and sets of accepted production and consumption bids. Typically, the market operator clears the market once a day. Additional markets for minor adjustments are possible on an hourly basis. Producers, consumers and the regulatory authority agree upon any market rules before their implementation.

In a monthly or yearly framework, the structure to allow competitive trade among producers and consumers is the floor for bilateral contracts. A bilateral contract is an agreement between a producer and a consumer so that the producer supplies electric energy to the consumer at a given price. Physical bilateral contracts are medium-term decisions lasting from one month to one year. Financial contracts to hedge price volatility are not considered in this work.

The power pool is commonly denominated Power Exchange (PX). Usually, producers are referred to as GENerating COmpanies (GENCOs) and consumers can be referred to as CONsumption COmpanies (CONCOs). Energy Service COmpanies (ESCOs) buy energy from bilateral contracts and from the power pool to sell it to different types of customers with the purpose of maximizing their own benefits. The market-clearing entity is often known as the Market Operator (MO). Power transactions are carried out through the transmission and distribution networks. The TRANSmission COmpanies (TRANSCOs) provide the wires to materialize the power transactions. Transmission companies are highly regulated entities that provide a non-discriminatory access to their wires for a regulated fee. Analogously, DIStribution COmpanies (DISCOs) offer a non-discriminatory use of their distribution wires for a regulated fee. The entity in charge of ensuring the technical feasibility of the power transactions agreed upon at the Power Exchange is denominated the Independent System Operator (ISO). The ISO 




Figure 1: Electricity market agents and structure.

has usually the authority to modify power transactions already scheduled if system security is at stake. These modifications should be minimal. The above structure is illustrated in Figure 1. Confusingly, sometimes the name ISO is applied to the MO in the technical literature and consumption and energy service companies are referred to as DISCOs. See Sheblé (1999) for additional details.

This paper addresses only energy markets. Although reserve and regulation markets are also relevant for the power industry, the largest share of market trade is within the energy market.

Relevant books addressing the new competitive framework include Sheblé (1999), Meier and Hobbs (1998), Chao and Huntington (1998), Ilic et al. (1998) and Hobbs et al. (2000). Dozens of conference papers on the subject can be found in the proceedings of the IEEE Power Engineering Society (http://www.ieee.org/) conferences, and the University of California Energy Institute symposia and workshops, in particular the Program on Workable Energy Regulation, POWER, (http://www.ucei.berkeley.edu). Relevant journal papers are mostly found in the issues of the "IEEE Transactions on Power Systems" (during the last ten years) and "The Electricity Journal", Elsevier Science, The Netherlands (http://www.elsevier.nl). 
The remainder of this paper is organized as follows. In Section 2 the producer perspective is adopted. Both bilateral contract and pool bidding issues are analyzed. Market power topics are also treated. In Section 3 the viewpoint of the energy service company is studied, and its decision-making problems are analyzed. In Section 4 the point of view of the consumer is considered for both short-term decisions and long-term ones. Section 5 presents the problems to be solved by the market operator. Three different market-clearing algorithms are reviewed: single-period auctions, multiperiod auctions and Walrasian auctions. Finally, Section 6 provides some conclusions.

\section{Producer viewpoint}

The decisions faced by a GENCO are described in this section. The decision making problems of a GENCO are mainly two: (i) how much energy to allocate to bilateral contracts and (ii) how much energy to sell in the pool. These problems are analyzed below.

\subsection{Bilateral contract selection}

In most electricity markets, producers and consumers are allowed to establish physical supply contracts outside the pool. From the point of view of a GENCO, it needs to determine if it would be more profitable to sell a certain amount of energy directly through one of these contracts or through the pool. As the main parameters in the contract (prices, quantities) are fixed in advance, a GENCO may reduce its risk significantly by using these contracts as an alternative to the pool. Consequently, any model that would consider these decisions must also take into account some representation of the risk associated with trading through the pool.

The decision problems associated with these bilateral contracts are of two (related) kinds: i) how to design a contract in an optimal manner, taking into account the peculiarities of the producer and the consumer, and ii) to decide if a contract with a given structure is of interest to the GENCO, as an alternative to the pool.

The details of a contract may vary significantly from one case to another. These details affect the specific structure of the mathematical models result- 
ing from the preceding decision problems. In what follows we will assume that a contract is a sequence through time of values of (demanded) energy satisfying certain constraints, and prices related to the energy amounts; these constraints and price functions define the contract. For example, a contract could be defined by a maximum amount of energy to be served in a given time (a year), with bounds on the energy served in each period within its time horizon, and prices depending on the total amount of energy served.

The following discussion will concentrate on the second decision problem, that is, to determine if a given contract is acceptable for a GENCO. The first problem (the design problem) could be solved in terms of this one, once some information on the form of the contract is available.

\subsubsection{Contract selection under uncertainty}

One of the main difficulties when posing and solving contract selection problems stems from the fact that the decisions that must be compared take place on quite different time frames. The outcome of the energy market (a daily process with hourly prices) must be compared to that of the bilateral contract under consideration (a monthly or yearly arrangement). Modeling these different time scales gives rise to problems of very large size.

One possible alternative is to summarize the expected behavior of the energy market through weekly or monthly averages. This works reasonably well if perfect competition is assumed, but it may not be appropriate if the GENCO has significant market power.

The presence of uncertainty, mostly associated with the prices resulting from the energy market, adds to the complexity of these models. Other possible sources of uncertainty are those derived from the effective use that will be made of the contract (that is, the specific amount of energy demanded), and the availability of renewable resources for energy generation. The particular (but important) case of hydrogeneration will be considered later on.

In summary, to decide if a contract would be acceptable for a GENCO, it would have to solve two optimization problems to compare the profits generated with the contract and those without it. If we assume the GENCO to be a price taker, each problem would maximize the profits from the pool 
and the contracts (with or without the one under consideration), subject to technical constraints and those specific for the contracts. It would have the form:

$$
\begin{aligned}
\max _{p_{i t}, \forall i, t ; s_{t}, \forall t} E_{\omega}\left\{\sum_{t}\left(\lambda_{t}(\omega) s_{t}-\sum_{i} c_{i t}\left(p_{i t}\right)\right)\right\} \\
\text { subject to } \quad \sum_{i} p_{i t}=s_{t}+\sum_{j} l_{j t}(\omega) \quad \forall t \\
p_{i t} \in \Pi_{i} \quad \forall i, t
\end{aligned}
$$

where $p_{i t}$ is the total power output of generator $i$ (owned by the GENCO) in period $t, s_{t}$ is the total power output of the GENCO that is allocated to the pool during period $t, c_{i t}\left(p_{i t}\right)$ is the production cost for energy generation $p_{i t}$ of generator $i$ at period $t$ (data), $\lambda_{t}(\omega)$ is the (average) value of energy market prices under realization of uncertainty $\omega$ for period $t$ (data), $l_{j t}(\omega)$ is the requirement of energy from contract $j$ during period $t$ under realization of uncertainty $\omega$ (data), $\Pi_{i}$ is the feasible operating region of generator $i$ (data), and $E_{\omega}\{\cdot\}$ indicates expected value over $\omega$. It should be noted that the above problem includes binary variables that do not appear explicitly in its formulation.

The optimal expected profit should be modified by the term $E_{\omega}\left\{\sum_{t} \sum_{j} \bar{\lambda}_{j t}(\omega) l_{j t}(\omega)\right\}$, where $\bar{\lambda}_{j t}(\omega)$ denotes the unit payment to be received from contract $j$ during period $t$. This value does not depend on the decision variables and can be taken into account after problem (2.1) has been solved.

Problem (2.1) maximizes the profits under the optimal allocation of generation between the energy market and bilateral contracts. The constraints are the operational restrictions on the units and the satisfaction of the contracts. The existence of contracts implies that the problem cannot be separated by generator or time unit, unless additional assumptions are made in advance.

The uncertainty is modeled through the energy market prices and the actual requirements of the contracts. Given the potential size of the problem, the periods usually considered are either weeks or months, and the energy market prices used in the model are averages over these time periods. The uncertainty is usually discretized by introducing scenarios for the parameters $\lambda_{t}(\omega)$ and $l_{j t}(\omega)$. The objective function could be modified 
to take into account the risk exposure of the GENCO, by adding terms penalizing this risk, for example.

In practice, this problem can be simplified by ignoring the uncertainty in the satisfaction of the contracts, and by making a priori assumptions on the allocation of generation from units to contracts.

Problem (2.1) is a large MINLP that can be approximated either as a MILP or as a continuous (and nonconvex) NLP. Its solution requires the use of either a sophisticated branch and cut solver (Brooke et al. (1998), GAMS (2000)) or a large scale NLP solver (Gill et al. (1997)).

\subsubsection{Renewable energies and water value functions}

The preceding model assumed a GENCO whose generating plants included only thermal units. Energy generated from hydroelectric units, if they are available, has very low production costs and it is a very valuable generation resource for the GENCO. The preceding model (2.1) must be modified to take into account that the availability of water in the reservoirs for the time horizon under consideration (several months to a few years) may introduce significant additional uncertainties, and that these reservoirs are typically interconnected within river basins.

Using model (2.1) as a reference, the modifications associated with the management of the hydro generation are: i) Hydro generation is a nonlinear function of water released and height in the reservoir, which may change significantly in the time horizon considered for these problems; this requires keeping track of both water released and water stored in the reservoirs. ii) The availability of water depends not only on the actions of the GENCO, but also on other stochastic parameters related to the climate, alternative uses for the water and the actions of the owners of other reservoirs in the basin. iii) The amount of water stored at the end of the planning period is an important decision variable, and should be treated explicitly in the model.

Regarding this last item, the storage of water in a given period, as opposed to its usage for hydro generation, allows to delay generation to other periods with larger expected unit profit. The water left in the reservoirs at the end of the planning horizon should be treated in this same manner, that is, it is a resource that should be stored if the expectation of future profits 
(beyond the planning horizon) is sufficiently large. As a consequence, an informed decision will require an estimation of these expected future profits. An efficient manner to do this is to introduce water value functions that quantify the expected future income for each amount of water stored at the end of the period in each reservoir.

The resulting model would have the form:

$$
\begin{aligned}
& \max _{p_{i t}, \forall i, t_{;}, \forall t ; x ; y} E_{\omega}\left\{\left(\sum_{i \in H} v_{i}\left(y_{i T}\right)+\sum_{t}\left(\lambda_{t}(\omega) s_{t}-\sum_{i \in G} c_{i t}\left(p_{i t}\right)\right)\right)\right\} \\
& \text { subject to } s_{t}+\sum_{j} l_{j t}(\omega)=\sum_{i} p_{i t} \quad \forall i, t \\
& p_{i t} \in \Pi_{i} \quad \forall i, t \in G \\
& p_{i t}=g_{i}\left(x_{i t}, y_{i t}\right) \quad \forall i, t \in H \\
& A x+B y=b(\omega)
\end{aligned}
$$

where $p_{i t}$ is the total power output of generator $i$ (owned by the GENCO) in period $t, s_{t}$ is the total output of the GENCO that is allocated to the pool during period $t, x_{i t}$ is the amount of water released through hydro unit $i$ (units in set $H$ ) in period $t$ ( $x$ is the vector of all $x_{i t}$ ), $y_{i t}$ is some average measure of the water stored in reservoir $i$ through period $t$ ( $y$ is the vector of all $\left.y_{i t}\right), v_{i}\left(y_{i T}\right)$ is the water value function (in monetary units) at reservoir $i$, evaluated at $y_{i T}$ (data), the water stored at the end of the planning horizon $T$ (data), $c_{i t}\left(p_{i t}\right)$ is the production cost of generator $i$ at period $t$ for the thermal generators (units in set $G$ ) (data), $g_{i}\left(x_{i t}, y_{i t}\right.$ ) provides the power generation at hydro unit $i$ corresponding to a water flow $x_{i t}$ and a storage level $y_{i t}$ (data), $\lambda_{t}(\omega)$ is the (average) value of energy market price under realization of uncertainty $\omega$ for period $t$ (data), $l_{j t}(\omega)$ is the requirement of energy from contract $j$ during period $t$ under realization of uncertainty $\omega$ (data), and $\Pi_{i}$ is the feasible operating region of generator $i$ (data). $A$ and $B$ are node-arc incidence matrices that represent the topology of the river basins where the reservoirs are located (if they are linked), as well as the dependence between time periods for the water stored in the reservoirs (data), and $b(\omega)$ is a vector of external inflows and outflows to the reservoirs (rain, evaporation and regulated flows) in the basins for uncertainty realization $\omega$ (data). It should be noted that the above problem includes binary variables that do not appear explicitly in its formulation.

The optimal expected profit should again be modified by 
$E_{\omega}\left\{\sum_{t} \sum_{j} \bar{\lambda}_{j t} l_{j t}(\omega)\right\}$, the expected income from the bilateral contracts, where $\bar{\lambda}_{j t}$ is the resulting price from contract $j$ during time period $t$.

To simplify the formulation we have assumed that each reservoir is associated with a single hydro unit. The main differences between this model and $(2.1)$ are the water value functions $v_{i}(\cdot)$ and the balances on flows and stored water in the reservoirs. If water levels in the reservoirs are assumed to be (approximately) constant, the variables representing the hydro units (water released and stored) can be replaced by the energy generated at these units, resulting in a model similar to that of a thermal unit, except that the hydro units have uncertain levels of availability.

Problem (2.2) requires an estimate of the water value functions $v_{i}(\cdot)$. This estimate can be obtained from data external to the model, or it can be generated within the model itself. An interesting and efficient proposal to compute an approximation for these functions within the model is given in Pereira and Pinto (1991). In it, model (2.2) is extended beyond the planning horizon, to cover for example several years. This extension must also incorporate the corresponding uncertain information for the additional periods. The resulting model is very large, but it is not solved directly; instead it is decomposed into the time periods corresponding to the original planning horizon and those beyond it. A Benders decomposition approach (Benders (1962)) is used to generate cuts from the subproblems corresponding to the periods beyond the planning horizon. These cuts provide piecewise linear approximations to the water value functions. The approximations are generated at the optimal values of the decision variables for a previous approximation, and the procedure is repeated until the error in the approximation of the water value function is below a certain tolerance. This procedure is closely related to the standard procedure in dynamic programming, where a so-called value function is approximated from its values at certain points, but in this case it is applied to the dual of the auxiliary problems; it is often referred to as dual dynamic programming.

The resulting model is a very large MINLP. It can be approximated by either MILP models (by introducing piecewise linear approximations to the functions in the model) or by large scale NLP models (by removing the zero-one variables). 


\subsection{Pool response}

A GENCO with no capability to alter market-clearing prices will schedule its production to maximize its profit given a forecasted price profile. Conversely, a GENCO with capability to alter market-clearing prices adjusts both (i) its productions and (ii) the resulting market-clearing prices to maximize its profit. The determination of an optimal adjustment requires a precise knowledge of how it can influence prices. This knowledge is embodied in the so-called price-quota (or residual demand) curve that provides the market-clearing price as a function of the GENCO market quota. Forecasting these price-quota curves is a challenging research topic.

Once the GENCO best production schedule is known, a bidding strategy to achieve this production schedule should be devised. This section will focus only on the determination of the GENCO best production schedule, which can be formulated as a mathematical programming problem.

For the sake of clarity, hydro units are not considered in the following. The models presented below can be extended to consider hydro units in an analogous fashion to the preceding description.

\subsubsection{Price taker}

A GENCO with no capability to alter market-clearing prices can be modeled as a number of generators that maximize their profits independently. In this case, given the market prices, the profit maximization problem for the GENCO as a whole decomposes directly by generator. Therefore, a single generator is considered in the following model. The objective of this generator is to maximize its profits subject to its operational constraints. Therefore, its profit maximization problem is formulated as:

$$
\begin{array}{ll}
\max _{p_{t}, \forall t} & \sum_{t}\left(\lambda_{t} p_{t}-c_{t}\left(p_{t}\right)\right) \\
\text { subject to } & p_{t} \in \Pi, \quad \forall t
\end{array}
$$

where $p_{t}$ is the energy produced by the generator at hour $t, c_{t}\left(p_{t}\right)$ is the production cost at hour $t$ (data), $\lambda_{t}$ is the forecasted market-clearing price at hour $t$ (data), and $\Pi$ is the feasible operating region of the generator (data). It should be noted that the above problem includes binary variables that do not appear explicitly in its formulation. 
The objective function of problem (2.3) includes two terms: revenues and costs. Their difference provides the profits for the GENCO. The constraints state that the generator should work within its feasible operating region. A detailed description of the operating region of a generator using MILP is provided in Arroyo and Conejo (2000).

The solution of this problem provides the optimal production of the generator every hour. The generator should bid in the market so that its optimal production plan is scheduled by the MO.

Model (2.3) is a MILP problem. Its size is small and it can be solved using a simple branch and bound solver.

\subsubsection{Price maker}

A GENCO with market power usually owns a significant number of generators. Its objective is to maximize its profit subject to the operation constraints of the generator. To that end, the GENCO modifies its hourly productions with the purpose of altering market-clearing prices to achieve the highest possible profits. This requires a coordinated action from all generators of the GENCO.

The above problem is formulated as:

$$
\begin{array}{ll}
\max _{p_{i t}, \forall i, t_{i}, \forall t} & \sum_{t}\left(\lambda_{t}\left(q_{t}\right) q_{t}-\sum_{i} c_{i t}\left(p_{i t}\right)\right) \\
\text { subject to } & p_{i t} \in \Pi_{i} \quad \forall i, t \\
& q_{t}=\sum_{i} p_{i t} \quad \forall t
\end{array}
$$

where $p_{i t}$ is the power output of generator $i$ (owned by the GENCO) at hour $t, q_{t}$ is the GENCO market quota at hour $t, c_{i t}\left(p_{i t}\right)$ is the production cost of generator $i$ at hour $t$ (data), $\lambda_{t}\left(q_{t}\right)$ is the GENCO price-quota function at hour $t$ (data) (Sheblé (1999)), and $\Pi_{i}$ is the feasible operating region of generator $i$ (data). It should be noted that the above problem includes binary variables that do not appear explicitly in its formulation.

The objective function of problem (2.4) represents the profits for the GENCO. The first block of constraints expresses the GENCO market quota as a function of the production of its generators. The second block of constraints enforces the operating restrictions of the generators belonging 
to the GENCO.

The solution of problem (2.4) provides the optimal production of every generator of the GENCO. The GENCO should bid in the market in such a way that its generators are allocated their optimal productions.

Problem (2.4) is a medium-size MINLP problem. Through the use of additional binary variables it can be converted into a MILP problem. Its solution requires the use of a sophisticated branch and cut solver.

\section{Energy service company viewpoint}

An energy service company obtains energy from bilateral contracts, from the pool and from its own production plants and sells it to different customers. The ESCO target is to maximize its own profit.

An ESCO must decide which are the most favorable bilateral contracts to sign in the medium term. In the short term, it buys in the pool any additional energy needed to supply its contractual obligations with its clients. If the ESCO has self-production capability, it can use it to protect itself against high prices in the pool. The contract selection and the pool operation problems are analyzed below.

\subsection{Bilateral contract selection}

For an ESCO, the choice of a portfolio of contracts is a similar problem to that of a GENCO, analyzed in Section 2.1. An important difference is that the ESCO must select both energy purchase and energy sales contracts.

The decisions on purchase and sales contracts involve both the design of the contracts and their evaluation versus alternatives (purchases from the pool). The remainder of the section will consider only the evaluation of alternatives. A model for this evaluation would estimate the profits associated with the optimal operation of the system under each of the alternative situations on a time horizon defined by the duration of the contract (typically 
one year). The model would have the following form:

$$
\begin{array}{cl}
\max _{\substack{p_{i t}, \forall i, t ; s_{t}, \forall t ; \\
b_{t}, \forall t ; r_{k t}, \forall k, t \\
\text { subject to }}} E_{\omega}\left\{\sum_{t}\left(\lambda_{t}(\omega)\left(s_{t}-b_{t}\right)-\sum_{i} c_{i t}\left(p_{i t}\right)-\sum_{k} \lambda_{k t}\left(r_{k t}\right) r_{k t}\right)\right\} \\
p_{i t} \in \prod_{i} \quad \forall i, t \\
r_{k t} \in \Gamma_{k}+\sum_{k} r_{k t}+b_{t} \quad \forall t
\end{array}
$$

where $p_{i t}$ is the total energy output of generator $i$ (owned by the ESCO) during period $t, s_{t}$ is the total energy output of the ESCO that is sold through the pool during period $t, b_{t}$ is the total amount of energy that the ESCO purchases through the pool during period $t, r_{k t}$ is the amount of energy that the ESCO purchases from contract $k$ in period $t, c_{i t}\left(p_{i t}\right)$ is the production cost of generator $i$ at period $t$ (data), $\lambda_{t}(\omega)$ is the (average) value of energy market price under realization of uncertainty $\omega$ for period $t$ (data), $l_{j t}$ is the amount of energy that the ESCO sells to contract $j$ in period $t$ (data), $\lambda_{k t}\left(r_{k t}\right)$ is the unit price associated with a purchase $r$ from contract $k$ during period $t$ (data), $\Pi_{i}$ is the feasible operating region of generator $i$ (data), and $\Gamma_{k}$ is the set of constraints associated with the specification of purchase contract $k$ (data). It should be noted that the above problem includes binary variables that do not appear explicitly in its formulation.

For simplicity, the preceding model has been formulated ignoring hydro generation. When comparing different alternatives, the optimal objective function should be modified by adding the term $E_{\omega}\left\{\sum_{t} \sum_{j} \bar{\lambda}_{j t}(\omega) l_{j t}(\omega)\right\}$, that is, the expected income from the bilateral contracts, independent of the variables. Note that $\bar{\lambda}_{j t}$ is the resulting price of contract $j$ during time $t$.

This model is a large scale MINLP that can be transformed into an large MILP (by adding binary variables for example). It can be solved using efficient branch and cut algorithms.

\subsection{Pool response and self-operation}

For simplicity, the energy allocated to bilateral contracts is not accounted for below. In this case, the ESCO target is to maximize its profits from the sale of energy to its consumers. This energy is either self-produced 
or bought in the pool. This profit maximization problem is formulated as:

$$
\begin{gathered}
\max _{p_{t}, \forall t ; b_{t}, \forall t}-\sum_{t}\left(c_{t}\left(p_{t}\right)+\lambda_{t} b_{t}\right) \\
\text { subject to } \quad p_{t}+b_{t}=d_{t} \quad \forall t \\
p_{t} \in \Pi
\end{gathered}
$$

where $p_{t}$ is the energy self-produced at hour $t, b_{t}$ is the energy bought from the pool at hour $t, d_{t}$ is the forecasted total customer demand at hour $t$ (data), $c_{t}\left(p_{t}\right)$ is the production cost of energy self-produced at hour $t$ (data), and $\lambda_{t}$ is the forecasted price of the energy bought in the pool at hour $t$ (data). It should be noted that the above problem includes binary variables that do not appear explicitly in its formulation.

The objective function of problem (3.2) includes two terms: costs from self-producing energy and costs from buying energy from the pool. After solving the problem, the objective function should be modified by adding a term that represents the revenues from selling energy to the customers, $\sum_{t} \alpha_{t} d_{t}$, where $\alpha_{t}$ is the customer selling price of the energy during hour $t$. This term does not depend on the optimization variables. The first block of constraints establishes that the customer demand should be supplied in every period. The last constraint requires that the generators belonging to the ESCO should work within its feasible operating region.

The solution of this problem provides the amount of power to buy from the pool and to self-produce in every period of the production horizon.

Problem (3.2) is a medium size MILP problem that can be easily solved using an efficient branch and cut solver.

\section{Consumer viewpoint}

The general case of a consumer with self-production capability is analyzed below. If the consumer has no self-production capability, the formulation below can be simplified in a straightforward manner. Two decision making problems faced by the consumer are addressed: (i) how much energy to buy from bilateral contracts and (ii) how much energy to buy from the pool. 


\subsection{Bilateral contract selection}

The objective of the $\mathrm{CONCO}$ in the medium-term horizon is to select the best bilateral contracts to sign among an array of available alternatives. If the bilateral agreements are adequate, the CONCO may decide not to buy from the pool. Conversely, if the portfolio of contracts is not competitive, the CONCO may decide to buy all its required energy from the pool.

The problem of a CONCO is similar to that of an ESCO, (3.1), except that the corresponding model would not include energy sales to other parties or to the pool.

The resulting model is:

$$
\begin{aligned}
\max _{\substack{p_{i t}, \forall i, t ; d_{t}, \forall t ; \\
b_{t}, \forall t ; r_{k t}, \forall k, t}} E_{\omega} & \left\{\sum_{t}\left(u_{t}\left(d_{t}\right)-\lambda_{t}(\omega) b_{t}-\sum_{i} c_{i t}\left(p_{i t}\right)-\sum_{k} \lambda_{k t}\left(r_{k t}\right) r_{k t}\right)\right\} \\
\text { subject to } & d_{t}=\sum_{i} p_{i t}+\sum_{k} r_{k t}+b_{t} \quad \forall t \\
p_{i t} & \in \prod_{i} \quad \forall i, t \\
r_{k t} & \in \Gamma_{k} \quad \forall k, t
\end{aligned}
$$

where $p_{i t}$ is the self-produced energy from generator $i$ (owned by the CONCO) during period $t, d_{t}$ is the total energy consumption of the CONCO during period $t, b_{t}$ is the total amount of energy that the CONCO purchases through the pool during period $t, r_{k t}$ is the amount of energy that the CONCO purchases from contract $k$ in period $t, c_{i t}\left(p_{i t}\right)$ is the production cost of generator $i$ at period $t$ (data), $u_{t}\left(d_{t}\right)$ is the CONCO utility function (in monetary units) at period $t$ (data), $\lambda_{t}(\omega)$ is the (average) value of energy market price under realization of uncertainty $\omega$ for period $t$ (data), $\lambda_{k t}\left(r_{k t}\right)$ is the unit price associated with a purchase $r$ from contract $k$ during period $t$ (data), $\Pi_{i}$ is the feasible operating region of generator $i$ (data), and $\Gamma_{k}$ is the set of constraints associated with the specification of purchase contract $k$ (data). It should be noted that the above problem includes binary variables that do not appear explicitly in its formulation.

The resulting model is again a large scale MINLP that can be solved by transforming it into a large MILP and using an efficient branch and cut algorithm. 


\subsection{Pool response}

From the pool perspective, the target of a $\mathrm{CONCO}$ is to maximize its consumer utility minus its self-production costs, subject to satisfying its own demand. For the sake of clarity, and without loss of generality, it is assumed that there is no bilateral contracts.

This model is formulated as:

$$
\begin{aligned}
\max _{p_{t}, \forall t ; b_{t}, \forall t} & \sum_{t}\left(u_{t}\left(d_{t}\right)-c_{t}\left(p_{t}\right)\right) \\
\text { subject to } \quad & b_{t}+p_{t}=d_{t} \quad \forall t \\
& p_{t} \in \Pi
\end{aligned}
$$

where $b_{t}$ is the energy bought in the pool by the consumer at hour $t, p_{t}$ is the energy self-produced by the consumer at hour $t, d_{t}$ is the CONCO own demand at hour $t$ (data),$u_{t}\left(d_{t}\right)$ is the consumer utility function at hour $t$ (data), and $c_{t}\left(p_{t}\right)$ is the consumer production cost at hour $t$ (data).

The objective function of problem (4.2) is the difference between the utility of the consumer and its self-production costs. The first block of constraints states that the demand of the consumer should be satisfied at every period. The last constraints establish that the generators owned by the consumer should work within their feasible operating region.

The solution of problem (4.2) provides the amounts of energy the consumer should buy from the pool or self-produce in each time period.

Problem (4.2) is a small-size MILP problem that is easy to solve.

\section{Pool operation viewpoint}

The market operator should clear the market using an appropriate procedure, agreed in advance by all market participants. Three market-clearing procedures are considered in this section:

1. Single-period auctions.

2. Multi-period auctions.

3. Walrasian auctions. 


\subsection{Single-period auctions}

The objective of a single-period auction is to maximize, for a single time period, the net social welfare subject to meeting the demand and the operating constraints of the producers. Therefore, periods are considered one at a time and inter-temporal constraints are neglected. As a result of ignoring these constraints, heuristics are needed to modify the auction solution in each time period, in order to ensure that it is technically feasible. To clear the market, 24 hourly auctions are carried out successively. This procedure is performed usually one day in advance. GENCOs, ESCOs and CONCOs submit their respective bids and the MO solves for every time period the problem below:

$$
\begin{aligned}
& \max _{d_{i}, \forall i ; p_{j}, \forall j} \sum_{i} \delta_{i} d_{i}-\sum_{j} \pi_{j} p_{j} \\
& \text { subject to } \quad 0 \leq p_{j} \leq \bar{p}_{j} \quad \forall j \\
& 0 \leq d_{i} \leq \bar{d}_{i} \quad \forall i \\
& \sum_{j \in m} p_{j} \in \Pi_{m} \quad \forall m \\
& \sum_{i \in n} d_{i} \in \Delta_{n} \quad \forall n \\
& \sum_{i} d_{i}=\sum_{j} p_{j}
\end{aligned}
$$

where $d_{i}$ is the demand bid $i, p_{j}$ is the production bid $j, \bar{d}_{i}$ is the size of demand bid $i$ (data), $\bar{p}_{j}$ is the size of production bid $j$ (data), $\delta_{i}$ is the price of demand bid $i$ (data), $\pi_{j}$ is the price of production bid $j$ (data), $\Delta_{n}$ is the feasible operating region of demand $n$ (data), $\Pi_{m}$ is the feasible operating region of producer $m$ (data), $i \in n$ indicates the set of demand blocks belonging to consumer $n$ (data), and $j \in m$ indicates the set of generation blocks belonging to producer $m$ (data). It should be noted that the above problem includes binary variables that do not appear explicitly in its formulation.

The objective function of problem (5.1) is the consumer surplus plus the producer surplus, i.e. the net social welfare. It is computed as the difference of two terms: the first term is the sum of accepted demand bids times their corresponding bidding prices; the second term is the sum of accepted 
production bids times their corresponding bidding prices. It should be noted that if the producers do not bid at their respective marginal costs, the second term of the objective function is not actually the producer surplus but the "declared" producer surplus. However, in this paper it will be considered, without loss of generality, that producers do bid at their actual marginal costs. The first block of constraints limits the sizes of the production bids. The second block of constraints specifies the sizes of the demand bids. The third block of constraints ensures that the set of bids from every producer should meet its production constraints. The fourth block of constraints enforces that the set of bids of every consumer should meet its consumption constraints. The fifth constraint states that the production should be equal to the demand, so that the market clears.

The solution of problem (5.1) provides the accepted production and demand bids and the market-clearing price, usually defined as the most expensive accepted production bid. Other definitions are also possible.

The above problem is a medium size MILP problem that can be easily solved.

\subsection{Multi-period auctions}

The objective of a multi-period auction is to maximize the net social welfare over the auction horizon subject to meeting, in every hour, the demand and the operation constraints of the producers. The same considerations on the net social welfare made for single-period auctions are also valid for multi-period ones. Inter-temporal constraints are explicitly taken into account (Arroyo and Conejo (2000)). GENCOs submit productions bids, ESCOs and CONCOs submit consumption bids and the MO solves the problem below:

$$
\begin{gathered}
\max _{d_{i t}, \forall i, t ; p_{j t}, \forall j, t} \sum_{t}\left(\sum_{i} \delta_{i t} d_{i t}-\sum_{j} \pi_{j t} p_{j t}\right) \\
\text { subject to } \quad 0 \leq p_{j t} \leq \bar{p}_{j t} \quad \forall j, t \\
0 \leq d_{i t} \leq \bar{d}_{i t} \quad \forall i, t \\
\sum_{j \in m} p_{j t} \in \Pi_{m} \quad \forall m, t
\end{gathered}
$$




$$
\begin{aligned}
& \sum_{i \in n} d_{i t} \in \Delta_{n} \quad \forall n, t \\
& \sum_{i} d_{i t}=\sum_{j} p_{j t} \quad \forall t
\end{aligned}
$$

where $d_{i t}$ is the demand bid $i$ at time $t, p_{j t}$ is the production bid $j$ at time $t, \delta_{i t}$ is the price of demand bid $i$ at time $t$ (data), $\pi_{j t}$ is the price of production bid $j$ at time $t$ (data), $\bar{d}_{i t}$ is the size of the demand bid $i$ at time $t$ (data), $\bar{p}_{j t}$ is the size of production bid $j$ at time $t$ (data), $\delta_{i t}$ is the price of demand bid $i$ at time $t$ (data), $\pi_{j t}$ is the price of production bid $j$ at time $t$ (data), $\Delta_{n}$ is the feasible operating region of demand $n$ (data), and $\Pi_{m}$ is the feasible operating region of producer $m$ (data). It should be noted that the above problem includes binary variables that do not appear explicitly in its formulation.

The objective function of problem (5.2) is the net social welfare over the whole planning horizon (consumer surplus plus producer surplus). The first block of constraints provides limits for production bids, while the second block limits demand bids. The third block of constraints establishes that the set of bids belonging to every producer should meet its production constraints. Analogously, the fourth block of constraints states that the set of bids of every consumer throughout the time horizon should meet its consumption constraints. The third and fourth blocks of constraints allow enforcing all types of inter-temporal constraints. The fifth block of constraints enforces the balance of production and demand in every period.

The solution of problem (5.2) provides the accepted production and demand bids and the market-clearing price in every time period. The marketclearing price in each hour is defined as the price of the most expensive accepted production bid that hour. Note that other definitions of marketclearing prices are possible.

The above problem is a large-scale MILP problem. A state-of-the-art branch and cut solver is required to solve it in a reasonable amount of time.

\subsection{Walrasian auctions}

A Walrasian auction (tatonnement) is a multi-round auction based on price modifications (Walras (1954), Galiana et al. (2000)). Note that previous auctions are not multi-round but just single-round. 
This auction is described in the steps below:

Step 1. The MO broadcasts hourly trial prices, $\lambda_{t}, \forall t$.

Step 2. Producers determine their productions to maximize their profits subject to their respective operation constraints. Therefore, each GENCO solves problem (2.3) or problem (2.4) and communicates to the MO the production schedule it is willing to carry out.

Step 3. ESCOs determine the energy to buy from the pool to maximize their respective profits. Thus, each ESCO solves problem (3.2) and informs the $\mathrm{MO}$ of its desirable consumption schedule.

Step 4. Consumers determine the amounts of energy that maximize their respective utilities. Therefore, each CONCO solves problem (4.2) and sends to the MO the consumption schedule it is willing to accept.

Step 5. The MO calculates hourly load imbalances.

Step 6. If hourly prices are unchanged in two consecutive rounds, they produce the market-clearing prices, and the auction stops; else the MO modifies prices aiming at balancing the load, broadcasts new hourly prices, and the auction continues in Step 2.

It should be noted that the above algorithm guarantees that each participant maximizes its individual profits. In fact, it corresponds to the Lagrangian relaxation solution of the dual problem of a centralized minimum cost operation problem (with perfect information). If this primal problem has a duality gap, the Walrasian auction may get trapped into an oscillatory behavior. If there is no duality gap, the Walrasian auction converges to the optimal solution of both the primal and dual problems. The oscillatory behavior is not so relevant in terms of the attained primal solution because it affects typically only a few units. However, changes in market-clearing price may be relevant. A challenging research problem is how to modify the original primal (cost minimization) problem so that its optimal solution does not change significantly but the duality gap is removed (Galiana et al. (2000)), and therefore it can be solved using a Walrasian auction. 


\section{Conclusions}

This paper reviews relevant mathematical programming problems that arise in a competitive electric energy framework, such as the ones arising in Europe and in many other places all over the world. The different perspectives of the producer, the consumer, the energy service company, and the pool operator are analyzed, and the associated mathematical programming problems are formulated and characterized. Many of the resulting models are large-scale MILPs. Improvements in the computation of solutions for these problems are of clear interest for the power industry. Other significant research challenges are related to:

- modeling decision making problems using stochastic programming, MILP and MINLP techniques,

- finding appropriate solution procedures, including decomposition techniques, and

- shortening required solution times.

\section{References}

Arroyo J.M. and Conejo A.J. (2000). Optimal Response of a Thermal Unit to an Electricity Market. IEEE Transactions on Power Systems 15, 1098-1104.

Benders J.F. (1962). Partitioning Procedures for Solving Mixed Variables Programming Problems. Numerische Mathematik 4, 238-252.

Brooke A., Kendrick D., Meeraus A. and Raman R. (1998). GAMS. A User's Guide, GAMS Development Corporation (http://www.gams.com/).

Chao H.-P. and Huntington H.G. (1998). Designing Competitive Electricity Markets. Fred Hillier's International Series in Operations Research \& Management Science. Kluwer Academic Publishers.

Galiana F.D., Motto A.L., Conejo A.J. and Huneault M. (2001). Decentralized Nodal-Price Self-Dispatch and Unit Commitment. In: The Next Generation of Unit Commitment Models. Fred Hillier's International Series in Operations Research \& Management Science. Kluwer Academic Publishers. 
GAMS Development Corporation (2000). GAMS - The Solver Manuals, GAMS Development Corporation (http://www.cplex.com/).

Gill P.E., Murray W. and Saunders M.A. (1997). User's Guide for SNOPT 5.3: a Fortran Package for Large-Scale Nonlinear Programming. Report NA 97-5, Department of Mathematics, University of California.

Hobbs B.F., Rothkopf M.H., O'Neill R.P. and Chao H.-P. (2001). The Next Generation of Unit Commitment Models. Fred Hillier's International Series in Operations Research \& Management Science. Kluwer Academic Publisher.

Ilic M.D., Galiana F.D. and Fink L.H. (1998). Power System Restructuring: Engineering and Economics. Kluwer Academic Publishers.

Meier P. and Hobbs B.F. (1998). Energy Decisions and the Environment - A Guide to the Use of Multicriteria Methods. Fred Hillier's International Series in Operations Research \& Management Science. Kluwer Academic Publishers.

Pereira M.V.F. and Pinto L.M.V.G. (1991). Multi-stage Stochastic Optimization Applied to Energy Planning. Mathematical Programming 52, 359-375.

Sheblé G.B. (1999). Computational Auction Mechanisms for Restructured Power Industry Operation. Kluwer Academic Publishers.

Walras L.M.-E. (1954). Éléments d'Économie Politique Pure; ou la Théorie de la Richesse Sociale. First Edition, 1874. English translation: Elements of Pure Economics or The Theory of Social Wealth, Homewood. Published for the American Economic Association and the Royal Economic Society, by R. D. Irwin. 